\title{
Urinary Excretion of Cyanidin Glucosides and Glucuronides in Healthy Humans After Elderberry Juice Ingestion
}

\author{
Roland Bitsch, ${ }^{1 *}$ Michael Netzel, ${ }^{1}$ Susanne Sonntag, ${ }^{1}$ Gabriele Strass, ${ }^{1}$ Thomas Frank, ${ }^{3}$ and Irmgard Bitsch ${ }^{2}$ \\ ${ }^{1}$ Institute of Nutritional Sciences, Friedrich-Schiller-University Jena, Dornburgerstrasse 29, 07743 Jena, Germany \\ ${ }^{2}$ Institute of Nutritional Sciences, Justus-Liebig-University Giessen, Wilhelmstrasse 20, 35392 Giessen, Germany \\ ${ }^{3}$ IMFORM GmbH International Clinical Research, Birkenweg 14, 64295 Darmstadt, Germany
}

Received 3 March 2004; revised 7 June 2004; accepted 15 June 2004

\begin{abstract}
In a pilot study with 6 females and 1 male, the metabolism of various cyanidin glucosides after oral administration of elderberry juice was investigated. The anthocyanin metabolites were detected in urinary excretion. After ingestion of a bolus quantity of $3.57 \mathrm{~g}$ total anthocyanins in a $150 \mathrm{~mL}$ elderberry juice concentrate, $0.053 \%$ of the administered dose was excreted in urine as glucosidically bound cyanidins within the first 5 hours. Only $0.003 \%$ of the ingested anthocyanin glucosides was excreted as cyanidin glucuronide, suggesting that this conversion step might be of minor importance in urinary excretion.
\end{abstract}

\section{INTRODUCTION}

Anthocyanins, as ubiquitous constituents of berries and coloured vegetables, are widespread in the plant dominion. Much attention has been paid in recent years to their radicals scavenging activity in vitro and in vivo and possible health benefits. The main anthocyanins being analysed in elderberries are various glucosylated cyanidins (Figure 1). Besides glucose, sambubiose is the typical sugar conjugate in elderberries $[1,2]$. Rate and extent of absorption, metabolisation, and excretion of elderberry anthocyanins are to date not fully understood. First of all, controversially discussed hitherto is whether also conjugated glucuronides of cyanidin are formed and excreted in vivo.

\section{STUDY DESIGN}

The study protocol was approved by the Ethics Committee of the Giessen University, Germany. After an overnight fasting, seven healthy nonsmoking volunteers (6 female and 1 male with a mean BMI of 21.5) consumed a bolus quantity of $150 \mathrm{~mL}$ of concentrated elderberry juice (containing $3.57 \mathrm{~g}$ of total anthocyanins) together with rolls and cheese. The elderberry juice concentrate was obtained from Wild (Heidelberg, Germany). Urine samples were collected initially and in hourly intervals over a period of 5 hours. The concentration of the excreted cyanidin conjugates was determined by HPLC analysis.

\section{SAMPLE EXTRACTION AND HPLC ANALYSIS}

All chemicals were purchased from Merck (Darmstadt, Germany). HPLC-DAD analyses of urinary samples ( $0-5$ hours after ingestion) were performed before and after hydrolysis of glucuronide conjugates with $\beta$ glucuronidase $[3,4,5,6]$. The anthocyanins were extracted by using a solid phase extraction cartridge (SepPakVac 12, Waters, Milford, Mass). The cartridge was first washed with $5 \mathrm{~mL}$ methanol and equilibrated with the same volume of $5 \%$ aqueous formic acid. Seven $\mathrm{mL}$ urine samples diluted with $2 \mathrm{~mL}$ formic acid were applied to the equilibrated cartridge. After washing with $5 \mathrm{~mL}$ of $5 \%$ formic acid, the anthocyanins were eluted with 5\% formic acid in $5 \mathrm{~mL}$ methanol. The eluate was evaporated to dryness in a vacuum rotary evaporator at $30^{\circ} \mathrm{C}$ and the extract redissolved with $200 \mu \mathrm{L}$ mobile phase before HPLC analysis. The chromatographic conditions adopted from Netzel et al [3] comprised separation on a Prontosil Eurobond RP-18 $(5 \mu \mathrm{m}, 250 \times 4 \mathrm{~mm}$ ID, Bischoff, Leonberg, Germany), protected by a guard column (LiChrospher $100 \mathrm{RP}-18,4 \times 4 \mathrm{~mm}$, Merck, Germany), and isocratic elution with water/formic acid/acetonitril (v/v/v) $=81 / 10 / 9$ (flow rate $0.5 \mathrm{~mL} / \mathrm{min}$ ) by using a high-precision pump (model L-6200, Merck-Hitachi, Darmstadt, Germany). The cyanidin glucosides were detected at $520 \mathrm{~nm}$ with the aid of a UV-VIS detector (L-4200, Merck-Hitachi).

Cyanidin glucosides excreted were identified by spiking blank urine samples with authentic compounds and comparing the retention time in the HPLC analysis and 
<smiles>[R2]Oc1cc2c(O[R1])cc(O)cc2[o+]c1-c1ccc(O)c(O)c1</smiles>

\begin{tabular}{lll}
\hline Anthocyanins & \multicolumn{1}{c}{ R1 } & \multicolumn{1}{c}{ R2 } \\
\hline Cyanidin 3-sam-5-gluc & Sambubiose & Glucose \\
Cyanidin 3, 5-digluc & Glucose & Glucose \\
Cyanidin 3-sam & Sambubiose & H \\
Cyanidin 3-gluc & Glucose & H \\
\hline
\end{tabular}

FIGURE 1. Chemical structures of elderberry anthocyanins.

TABLE 1. Urinary excretion of anthocyanins as unchanged glucosides and glucuronides after elderberry juice consumption (means \pm $\mathrm{SD})$.

\begin{tabular}{|c|c|c|c|c|c|}
\hline \multirow{2}{*}{ Anthocyanins } & \multirow{2}{*}{$\begin{array}{c}\text { Doses } \\
\text { (mg/subject) }\end{array}$} & \multicolumn{2}{|c|}{ Total excretion (glycosides + glucuronides) } & \multicolumn{2}{|c|}{ Glucuronide excretion } \\
\hline & & $(\mathrm{mg} / 5 \mathrm{~h})$ & $(\%)^{2}$ & $(\mathrm{mg} / 5 \mathrm{~h})$ & $(\%)^{2}$ \\
\hline cya-3, 5-digluc $^{1}$ & 215.0 & $0.313 \pm 0.227$ & $0.145 \pm 0.105$ & $0.032 \pm 0.021$ & $0.015 \pm 0.010$ \\
\hline cya-3-sam & 2245.8 & $0.962 \pm 0.521$ & $0.043 \pm 0.023$ & $0.045 \pm 0.024$ & $0.002 \pm 0.001$ \\
\hline cya-3-gluc & 1108.2 & $0.601 \pm 0.321$ & $0.054 \pm 0.029$ & $0.038 \pm 0.025$ & $0.003 \pm 0.002$ \\
\hline sum & 3569.0 & $1.876 \pm 1.063$ & $0.053 \pm 0.030$ & $0.116 \pm 0.049$ & $0.003 \pm 0.001$ \\
\hline
\end{tabular}

${ }^{1}$ Amounts of cya-3-sam-5-gluc and cya-3, 5-digluc were calculated as cya-3, 5-digluc.

${ }^{2}$ Calculated as the ratio of amounts excreted (within $5 \mathrm{~h}$ ) to amounts/doses ingested.



FIGURE 2. Time-course plots (mean $\pm \mathrm{SD}$ ) of total anthocyanins (glucosides + glucuronides) and anthocyanin glucuronides in human urine following ingestion of $150 \mathrm{~mL}$ of elderberry juice.

UV-visible spectra. Standard curves were prepared for quantification prior to the preparation procedure. The detection limit $(\mathrm{S} / \mathrm{N} \geq 10)$ was between $0.13 \mathrm{ng}$ and $0.60 \mathrm{ng} /$ injection volume $(100 \mu \mathrm{L})$. Aliquots of each urine sample were acidified with formic acid $(2 \mathrm{~mL} / 0.2 \mathrm{~mL})$ and stored frozen at $-80^{\circ} \mathrm{C}$ until analysis.

\section{RESULTS AND DISCUSSION}

The analysed anthocyanin conjugates in urine are shown in Table 1. The urinary excretion of total antho- cyanins (unchanged cyanidin glucosides and their glucuronide conjugates) within 5 hours was $0.053 \pm 0.030 \%$ of the administered dose. Only $6.2 \pm 2.2 \%$ of the excreted amount consisted of glucuronides. Based on this recovery, the percentage of glucuronides in urinary excretion was $0.003 \pm 0.001 \%$ (calculated as the ratio of anthocyanin glucuronides excreted to anthocyanin glucosides ingested). The excretion pattern of total anthocyanins in Figure 2 demonstrates that a maximal excretion rate is reached 1 hour after intake, followed by a rapid drop to initial values about 5 hours after intake, resembling a first-order excretion kinetics.

The metabolic conversion of anthocyanins within the organism remains to be elucidated. In principle, glucuronidation or conjugation with sulphuric acid is a common final metabolic step to facilitate urinary excretion. Various metabolic conversion products of anthocyanins were found by several authors. Tsuda et al [7] found no cyanidin glucuronides in livers and kidneys of rats after oral administration of cyanidin 3-glycoside, but cyanidin was converted to peonidin and protocatechuic acid.

A different situation seems to exist to date in man. In the urinary excretion of elderly women, minor amounts of glucuronides of peonidin and cyanidin 3-glucoside could be deteced in half of the volunteers besides unchanged glucosides after elderberry consumption, which does correspond to our results $[2,5]$. Only glucosides of elderberry anthocyanins, however, could be found in plasma and urine of volunteers by other authors $[8,9]$. 


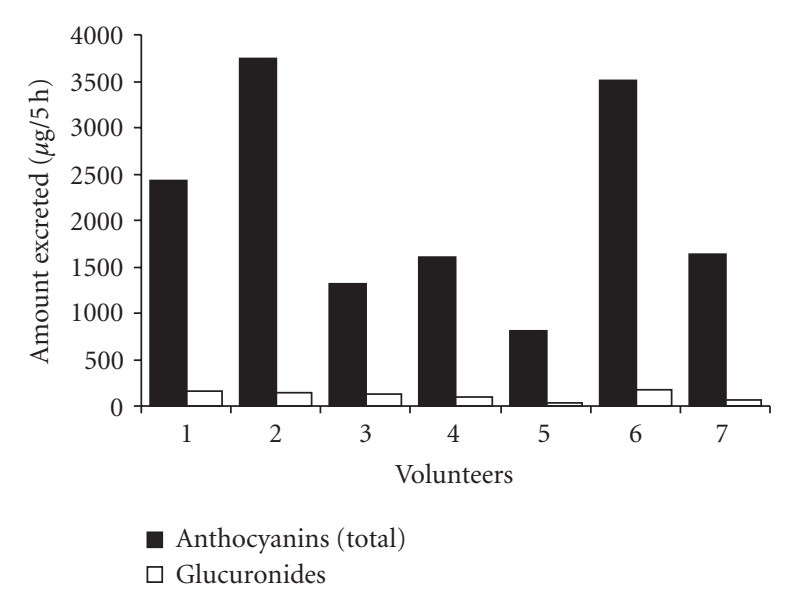

Figure 3. Individual amounts excreted of total anthocyanins (glucosides + glucuronides) and anthocyanin glucuronides within 5 hours following ingestion of $150 \mathrm{~mL}$ of elderberry juice.

To entirely estimate the extent of glucuronidation of anthocyanins as metabolic fate, it has to be considered that besides urinary excretion, biliary secretion may also serve as a possible way of elimination, particularly known for glucuronides. Newer studies, however, revealed that after intake of elderberry extract, the identical pattern of glucosylated cyanidins could be detected in plasma as in urine [10]. Thus, the results of the quantification of excretion products in the present study demonstrate that, at least in the given dose range, glucuronidation of cyanidin obviously represents a negligible conversion step in the metabolism of cyanidin ingested from elderberries. The proportion of glucuronide conjugates seems to represent a rather constant but very small proportion despite interindividual differences of total anthocyanin excretion (Figure 3). This may be in contrast to other fruits such as strawberry anthocyanins, being predominantly excreted in urine as glucuronides besides small amounts of sulfoconjugates [11]. It remains to show the extent to which the administered dose level does determine the site of metabolism. There exists some body of evidence that large doses of polyphenols are primarily metabolised in the liver, whereas small doses may be metabolised by the intestinal mucosa, with the liver playing a secondary role to further modify the polyphenol conjugates [12].

\section{REFERENCES}

[1] Murkovic M, Toplak H, Adam U, Pfannhauser W. Analysis of anthocyanins in plasma for determination of their bioavailability. Journal of Food Composition and Analysis. 2000;13:291-296.

[2] Cao G, Muccitelli HU, Sanchez-Moreno C, Prior RL. Anthocyanins are absorbed in glycated forms in elderly women: a pharmacokinetic study. Am J Clin Nutr. 2001;73(5):920-926.

[3] Netzel M, Strass G, Janssen M, Bitsch I, Bitsch R. Bioactive anthocyanins detected in human urine af- ter ingestion of blackcurrant juice. J Environ Pathol Toxicol Oncol. 2001;20(2):89-95.

[4] Bub A, Watzl B, Heeb D, Rechkemmer G, Briviba K. Malvidin-3-glucoside bioavailability in humans after ingestion of red wine, dealcoholized red wine and red grape juice. Eur J Nutr. 2001;40(3):113-120.

[5] Wu X, Cao G, Prior RL. Absorption and metabolism of anthocyanins in elderly women after consumption of elderberry or blueberry. $J$ Nutr. 2002;132(7):1865-1871.

[6] Andlauer W, Stumpf C, Frank K, Furst P. Absorption and metabolism of anthocyanin cyanidin-3glucoside in the isolated rat small intestine is not influenced by ethanol. Eur J Nutr. 2003;42(4):217-223.

[7] Tsuda T, Horio F, Osawa T. Absorption and metabolism of cyanidin 3-O-beta-D-glucoside in rats. FEBS Lett. 1999;449(2-3):179-182.

[8] Milbury PE, Cao G, Prior RL, Blumberg J. Bioavailablility of elderberry anthocyanins. Mech Ageing Dev. 2002;123(8):997-1006.

[9] Mulleder U, Murkovic M, Pfannhauser W. Urinary excretion of cyanidin glycosides. J Biochem Biophys Methods. 2002;53(1-3):61-66.

[10] Bitsch I, Janssen M, Netzel M, Strass G, Frank T. Bioavailability of anthocyanidin-3-glycosides following consumption of elderberry extract and blackcurrant juice. Int $\mathrm{J}$ Clin Pharmacol Ther. 2004;42(5):293-300.

[11] Felgines C, Talavera S, Gonthier MP, et al. Strawberry anthocyanins are recovered in urine as glucuro- and sulfoconjugates in humans. J Nutr. 2003;133(5):1296-1301.

[12] Scalbert A, Williamson G. Dietary intake and bioavailability of polyphenols. J Nutr. 2000;130(8S suppl):2073S-2085S.

* Corresponding author.

E-mail: roland.bitsch@uni-jena.de

Fax: +49 3641 949632; Tel: +49 3641949630 

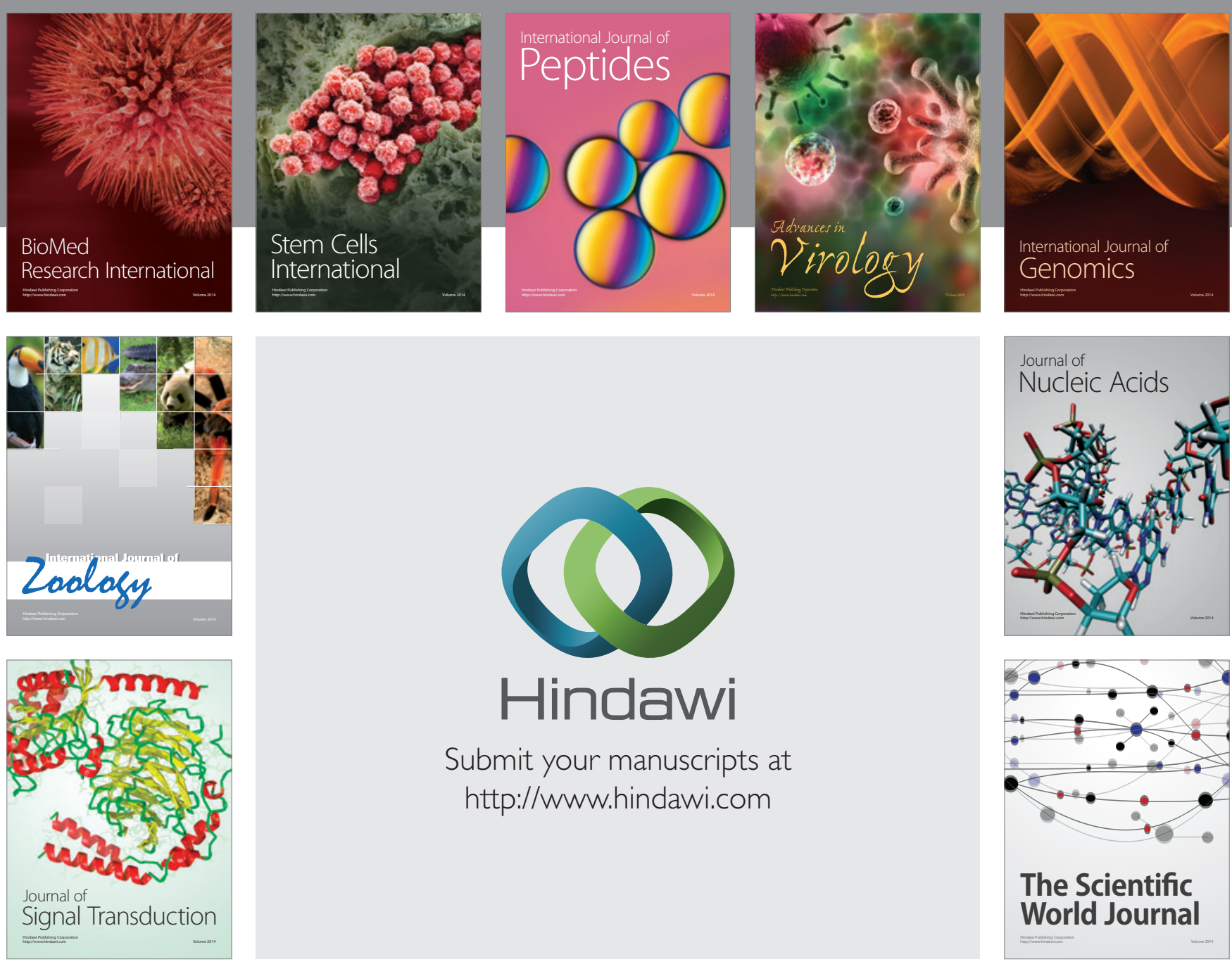

Submit your manuscripts at

http://www.hindawi.com
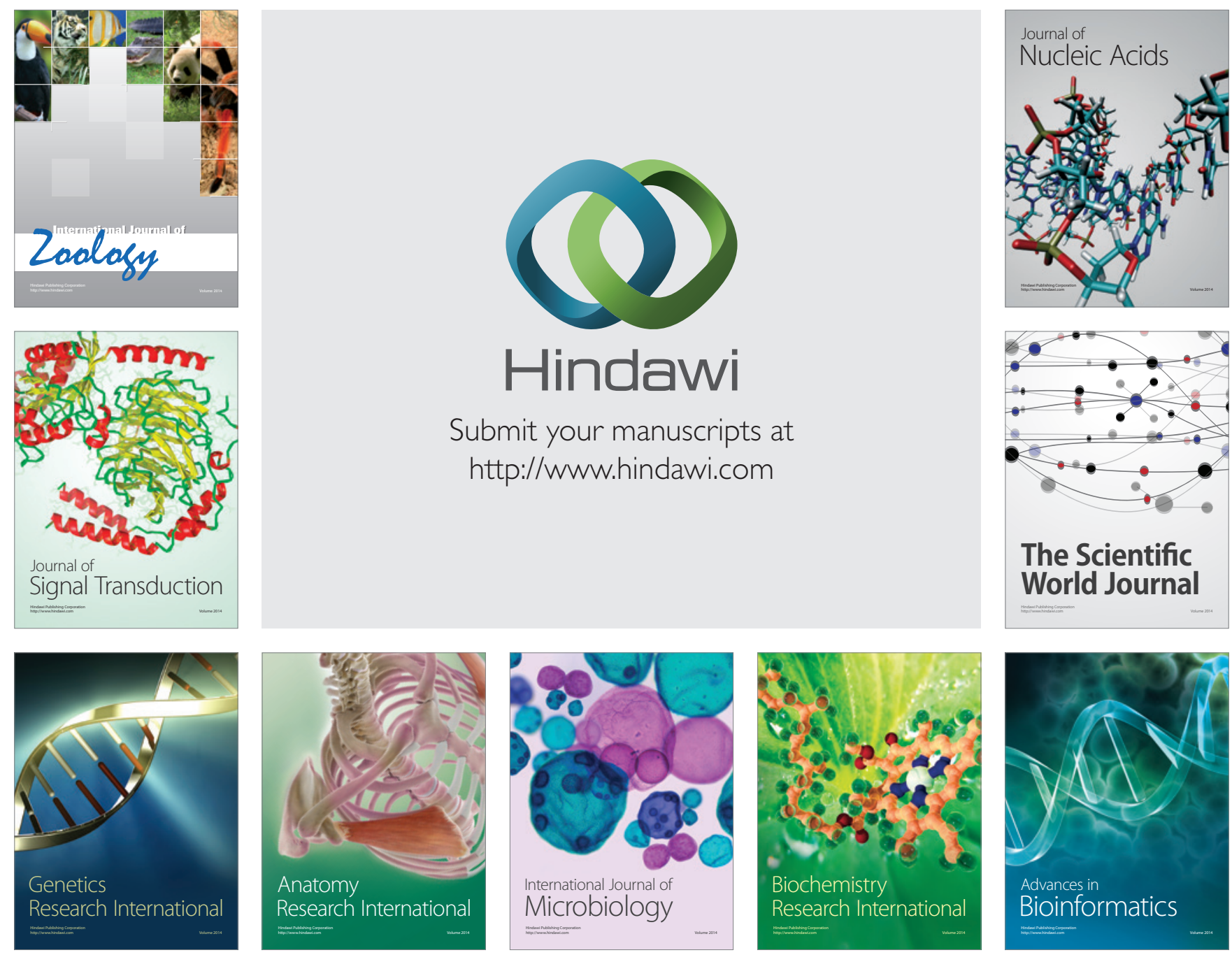

The Scientific World Journal
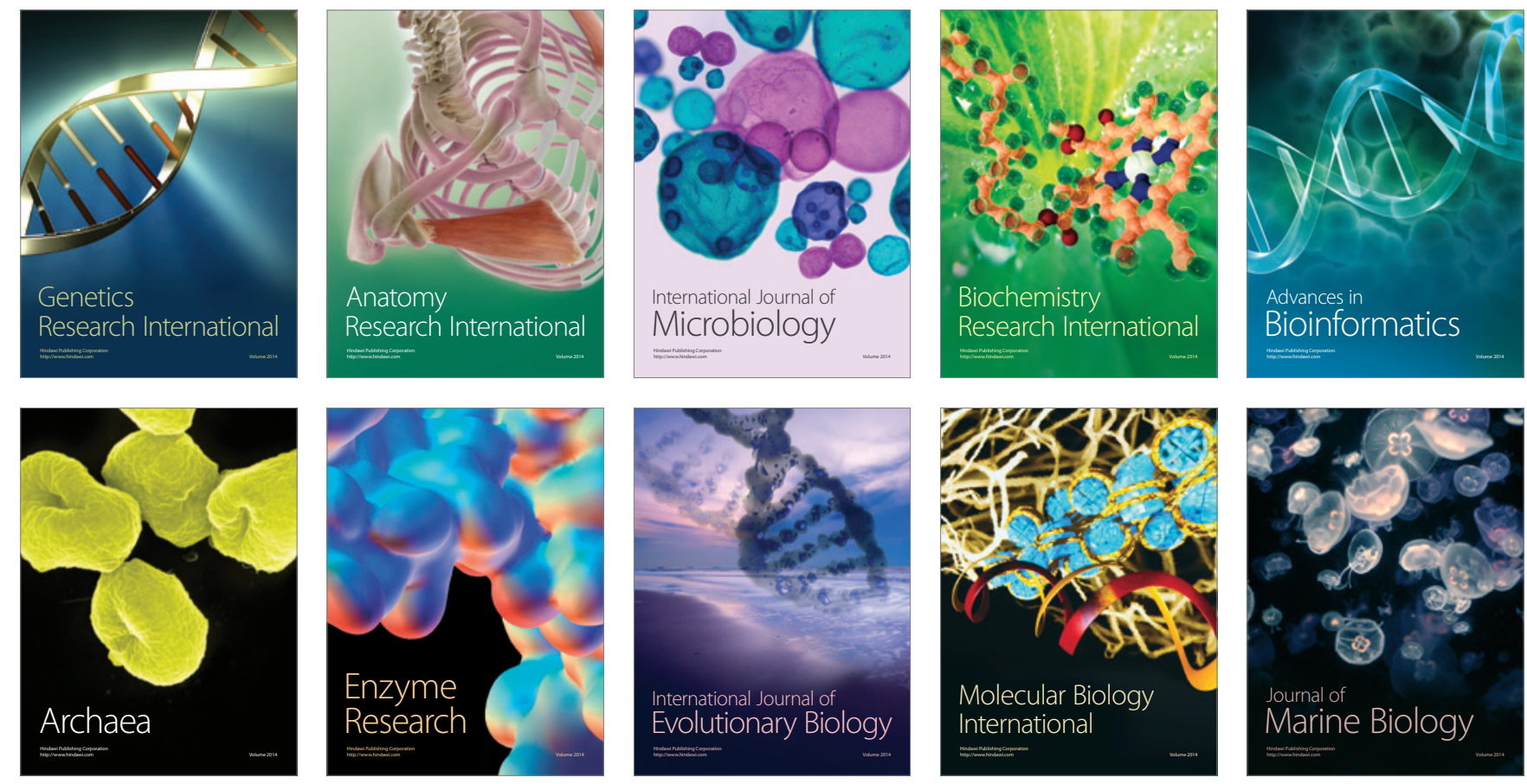